\title{
La Red de Investigadores sobre Cine Latinoamericano (RICiLa): cartografía de las problemáticas abordadas en el último lustro
}

Javier Cossalter ${ }^{1}$

${ }^{1}$ Doctor de la Universidad de Buenos Aires, área Historia y Teoría de las Artes. Pos-doctor en Ciencias Humanas y Sociales por la Universidad de Buenos Aires. Investigador Asistente del del Consejo Nacional de Investigaciones Científicas y Tecnológicas (CONICET). Docente de la cátedra de Introducción al Cine y a las Artes Audiovisuales en la carrera de Artes, Facultad de Filosofía y Letras, Universidad de Buenos Aires. Secretario general de la Red de Investigadores sobre Cine Latinoamericano (RICiLa) y miembro del Centro de Investigación y Nuevos Estudios sobre Cine (ClyNE). 


\section{Resumen}

La Red de Investigadores sobre Cine Latinoamericano fue creada con el propósito de conformar un circuito de intercambio de conocimientos y fomentar los estudios acerca del cine de la región. El presente trabajo intenta realizar un mapeo en torno a los proyectos de investigación de los miembros de la Red con el objetivo de dar a conocer el panorama actual acerca del contexto institucional de producción de estos trabajos, así como desentrañar las problemáticas histórico-analíticas y los enfoques de aproximación abordados en los mismos. A través del análisis comparado este ensayo permite observar una preponderancia de las universidades públicas como entes promotores de la investigación sobre cine latinoamericano, la focalización en cinematografías nacionales en estrecho vínculo con la procedencia institucional, la comprensión del cine de la región en tanto fenómeno global y el reconocimiento de una multiplicidad de perspectivas de acercamiento al objeto cinematográfico que van del análisis textual hasta el estudio de la recepción.

Palabras clave: cine latinoamericano; redes y asociaciones; proyectos de investigación.

\section{Resumo}

A Rede de Pesquisadores sobre Cinema Latino-Americano foi criada com o objetivo de formar um circuito de troca de conhecimento e promover estudos em torno do cinema da região. O presente trabalho procura fazer um mapeamento em torno dos projetos de pesquisa dos membros da Rede, a fim de apresentar o panorama atual sobre o contexto institucional de produção dessas obras, bem como desvendar os problemas histórico-analíticos e as abordagens de aproximação abordada neles. Por meio de análise comparativa, este ensaio permite observar uma preponderância das universidades públicas como promotoras da pesquisa cinematográfica latino-americana, o reparo em cinematografias nacionais em estreita conexão com a proveniência institucional, a compreensão do cinema da região como um fenômeno global e o reconhecimento de uma multiplicidade de perspectivas de abordagem ao objeto cinematográfico que vão da análise textual ao estudo da recepção.

Palavras-chave: cinema latino-americano; redes e associações; projetos de pesquisa

\section{Abstract}

The Red de Investigadores sobre Cine Latinoamericano was created with the purpose of forming a circuit of exchange of knowledge and to promote studies on the cinema of the region. This work tries to map the research projects of the members of the Network with the proposal of announcing the current panorama about the institutional context of production of these works as well as to unravel the historical-analytical problems and the approaches addressed in them. Through comparative analysis, this essay allows us to observe a preponderance of public universities as promoters of Latin American film research, the focus on national cinematographies linked with the institutional origin, the comprehension of regional cinema as a global phenomenon and the recognition of a multiplicity of perspectives of approach to the cinematographic object that goes from textual analysis to the study of reception.

Keywords: Latin American Cinema; Networks and Associations; Research Projects 


\section{Introducción}

La Red de Investigadores sobre Cine Latinoamericano (RICiLa) fue gestada a través de una iniciativa propuesta en el Proyecto de Investigación CeALCl financiado por la Fundación Carolina de España en 2011 - titulado Representación y revolución en el cine latinoamericano del período clásico-industrial: Argentina, Brasil, México - en el cual intervinieron especialistas de diversas nacionalidades. El objetivo principal consistía en conformar un circuito de producción e intercambio de conocimiento entre aquellos investigadores abocados al cine de América Latina que residieran tanto dentro como fuera de la región, junto con la promoción y el incentivo de perspectivas de análisis fílmico comparado. De este modo, la Red comenzó a funcionar a fines del 2011 y fue presentada públicamente en Buenos Aires durante el I Simposio Iberoamericano de estudios comparados sobre cine: representaciones de los procesos revolucionarios en el cine argentino, brasileño y mexicano, organizado por el Centro de Investigación y Nuevos Estudios sobre Cine (ClyNE) perteneciente a la Facultad de Filosofía y Letras de la Universidad de Buenos Aires. Al momento de su fundación la misma contó con la participación de setenta y cinco miembros de catorce países diferentes. En la actualidad, y luego de ocho años de vida, RICiLa alberga a doscientos cuarenta investigadores distribuidos en veintiocho países, ${ }^{2}$ lo que indica un notable crecimiento no sólo a nivel institucional, sino también en lo que respecta al marcado interés que fue cobrando el cine latinoamericano como objeto de estudio.

Desde su creación la Red dispone de una página web donde cada miembro puede actualizar su perfil académico además de difundir su producción escrita ponencias, artículos, capítulos y libros - e informar sobre múltiples convocatorias de trabajos en torno al campo de los estudios cinematográficos. A su vez, la web de RICiLa permite alojar toda índole de materiales como por ejemplo conferencias filmadas, catálogos y otro tipo de publicaciones digitales. Asimismo, la Red ha impulsado la participación activa de sus miembros a partir de la concreción de algunas actividades específicas. Entre ellas, es posible señalar la co-organización del II Simposio Iberoamericano de estudios sobre cine y audiovisual: perspectivas interdisciplinarias. Debates del cine y de la historia (2012) - en donde asistieron expositores de Argentina, Brasil, Chile, Uruguay y México - y del III Simposio Iberoamericano de estudios comparados: prácticas y relaciones transnacionales en el cine latinoamericano silente y clásico (2016) - con la presencia de los conferencistas Julia Tuñón y Eduardo Morettin; la publicación en formato de libro digital de las Actas del II Simposio (2013) y del I

\footnotetext{
${ }^{2}$ Estos son: Alemania, Argentina, Austria, Bélgica, Bolivia, Brasil, Canadá, Chile, Colombia, Costa Rica, Cuba, Ecuador, España, Estados Unidos, Francia, Grecia, Hong Kong, Inglaterra, Irlanda, Israel, México, Paraguay, Perú, Puerto Rico, República Checa, República Dominicana, Uruguay y Venezuela
} 
Simposio (2015); ${ }^{3}$ la publicación de un Dossier monográfico acerca del cortometraje documental latinoamericano en los años sesenta y setenta (2015) - compilación que articuló escritos sobre las cinematografías de Argentina, Bolivia, Brasil, Chile, Cuba, México, Perú, Uruguay y Venezuela; ${ }^{4}$ y el desarrollo de filmografías comentadas en torno a cada una de las cinematografías latinoamericanas, que contemplen los films más destacados de cada país - proyecto iniciado en 2018 y todavía en curso.

Por otra parte, a fines del año 2013, con la intención de acercar entre sí y afianzar los vínculos entre los investigadores de la Red, se propuso que cada integrante describa y comente los proyectos -individuales y/o colectivos- de los cuales formara parte. Resultaba - y hoy en día más aún - de suma importancia e interés conocer el amplio arco de tópicos y problemáticas relacionadas con el cine latinoamericano escogidas por los miembros en cada rincón del planeta. Si bien no todos los que participan de la Red efectivamente están involucrados en proyectos de investigación acreditados, el material hasta ahora recabado da cuenta de un panorama parcial, pero no por ello despreciable, acerca de los estudios sobre cine en América Latina durante los últimos seis años. ${ }^{5}$ Este es, en suma, el disparador que motiva el presente informe. La propuesta consiste entonces en realizar una cartografía de los proyectos de investigación - culminados o en curso - efectuados por los miembros de RICiLa desde su fundación hasta la fecha, con el interés de evaluar las líneas temáticas y metodológicas de abordaje así como también dar cuenta del marco contextual en el que estos se desenvuelven. La organización del trabajo estará atravesada por el método del análisis comparado, trasladando este enfoque de aproximación del objeto cine al abordaje de los estudios sobre cine. De este modo podremos reconocer "similitudes y diferencias (...) mediante patrones de comparación precisos, acordes a contextos históricos específicos y a preocupaciones puntuales" (LUSNICH, 2011: 37).

Por tal motivo, dividiremos el escrito en tres secciones. En la primera, pondremos el foco de atención en las instituciones que financian y albergan estos proyectos. En la segunda parte haremos un comentario sobre el origen nacional de las cinematografías seleccionadas y los períodos investigados. Finalmente, el tercer apartado estará reservado para discutir acerca de los problemas y objetos de estudio contemplados en las diversas investigaciones. En este punto se intentará hacer dialogar a los diferentes planteos tratando de establecer perspectivas de análisis comunes y

\footnotetext{
${ }^{3}$ Ambas fueron editadas por la Secretaría de Publicaciones de la Facultad de Filosofía y Letras de la Universidad de Buenos Aires.

${ }^{4}$ El mismo fue presentado en la edición número doce de la revista académica digital Imagofagia.

${ }^{5}$ Los resúmenes de dichos proyectos se encuentran disponibles para su consulta en la página web de RICiLa, http://www.ricila.com/category/PIndividuales.
} 
horizontes particulares. Por último, la conclusión brindará una reflexión en relación al desarrollo y devenir de los estudios fílmicos sobre América Latina.

Filiación y pertenencia institucional: ¿Cuáles son las entidades que financian las investigaciones sobre el cine de América Latina?

De acuerdo al relevamiento realizado, las universidades y los organismos científicos son las principales fuentes de sustento y apoyo al estudio sobre el cine latinoamericano. En un segundo escalón, muy por detrás, se ubican las cinetecas, cinematecas, fundaciones y asociaciones. En último lugar se encuentra la producción independiente de conocimiento. Con respecto al primer rango sobresalen los entes estatales - nacionales o federales. Y dentro de este, Brasil es el país que exhibe una mayor variabilidad de opciones. En relación al ámbito científico figuran el Conselho Nacional de Desenvolvimento Científico e Tecnológico (CNPq) y la Fundação de Amparo à Pesquisa do Estado de São Paulo (FASEP), que cuentan con más de sesenta y cincuenta años de funcionamiento, respectivamente. En torno al campo educativo y universitario, la Universidade de São Paulo alberga tres investigaciones mientras que la Universidade Federal Fluminense radica a dos. Luego, aparece la Universidade Federal da Bahia, la Universidade Estadual de Campinas y la Universidade Regional Integrada do Alto Uruguai e das Missões con un proyecto cada una. En Argentina, el Consejo Nacional de Investigaciones Científicas y Tecnológicas (CONICET) - cuya fundación se remonta hacia fines de los años cincuenta - es la única entidad de financiamiento destinada únicamente al sostén de la Ciencia y aparece en al menos seis oportunidades junto al respaldo de la Universidad de Buenos Aires (UBA). La Universidad Nacional de General Sarmiento (UNGS) y la Universidad Nacional de San Martín (UNSAM) emergen igualmente como espacios de formación y contención para el investigador en cine. El tercer lugar lo ocupa México con cuatro proyectos de tres entidades universitarias diferentes: dos en la Universidad Autónoma de Nuevo León, uno en la Universidad Autónoma de Baja California y uno en la Universidad de Guadalajara. Colombia y Venezuela presentan asimismo alternativas nacionales y públicas. Se trata de la Universidad Nacional de Colombia y la Universidad Pedagógica Nacional, por un lado, y de la Universidad Pedagógica Experimental Libertador (UPEL) junto a la Universidad Central de Venezuela, por el otro. Finalmente, en Bolivia, la principal universidad pública del país -la Universidad Mayor de San Andrés- tiene también un lugar entre las instituciones educativas de fomento a la investigación. Como nota destacada en relación a este sector vale mencionar a las dos únicas universidades públicas por fuera del continente latinoamericano que amparan este tipo de trabajos: Universität Wien (Universidad de Viena) y University of Memphis. Ahora bien, alrededor del campo 
universitario privado sólo se han contemplado dos casas de estudio, y una de ellas es extranjera: New York University. La Pontifícia Universidade Católica do Rio de Janeiro es entonces - hasta el momento y respecto al balance parcial realizado entre los miembros de RICiLa - la única institución universitaria latinoamericana no pública que respalda la investigación sobre cine.

En cuanto a la segunda línea de apoyo a la investigación contamos con organismos de diversa jerarquía, aunque todos dedicados íntegramente al estudio del séptimo arte. En este sentido, podemos señalar el aporte de la Cineteca de la Universidad de Chile - que bien podría figurar en el rango anterior por depender de una secretaría universitária -, la Cinemateca Dominicana, la Asociación Civil Cine 100 \% Venezolano y la Fundación FAMICINE, también de Venezuela. Finalmente, hallamos un solo proyecto sin pertenencia institucional, es decir, desarrollado de modo autogestionado e independiente.

En este sentido, y luego de este breve recorrido, la primera conclusión que aflora es la preponderancia casi absoluta de la universidad estatal y pública - primordialmente dentro de los confines de la región - en tanto ente de sustento para los proyectos sobre cine latinoamericano. A su vez, cabe advertir la diversificación dentro del terreno universitario, tanto en el ámbito regional como a nivel nacional.

\section{Marco territorial y temporal: ¿Qué cinematografías predominan en los proyectos y qué segmentos de la historia del cine prevalecen?}

Una de las interrogantes iniciales que surgió en el momento de acercarse a los proyectos, además de vislumbrar cuáles son los cines más escogidos, fue el siguiente: ¿los investigadores trabajan exclusivamente las cinematografías del país donde están radicados sus estudios? La respuesta no es lineal y directa pero pueden apreciarse algunas particularidades. En principio, es posible distinguir a los proyectos en donde se explora una sola cinematografía de aquellos en los cuales intervienen cines de diferentes nacionalidades. Entre los primeros, el cine de Argentina aparece en cinco oportunidades, el de México y el de Chile en cuatro, el de Bolivia en dos, el de Brasil y el de Colombia en una. En cuanto al origen de estas investigaciones el resultado no es homogéneo. Tanto en los casos del cine argentino como del mexicano, todos los proyectos, excepto uno, son con sede local mientras que con respecto a Chile la mitad de los mismos son externos al país andino, e incluso se encuentran en desarrollo por fuera de América Latina. En Bolivia, uno de los dos proyectos es externo. Finalmente, los trabajos sobre Brasil y Colombia pertenecen a instituciones de dichas naciones. En este punto, dos casos merecen una mención aparte. Por un lado, la cinematografía de Venezuela hace su incursión en seis proyectos, llevados a cabo en su totalidad por investigadores locales 
y contemplando al cine venezolano como único objeto de estudio. Por otro lado, República Dominicana sólo figura en un proyecto que, de hecho, no está enfocado rigurosamente en el cine de su país sino en la historia de su Cinemateca.

En relación a los trabajos que articulan más de una cinematografía, la preponderancia varía así como también se percibe una heterogeneidad en el origen de los mismos. El cine argentino se manifiesta dos veces - ninguno de los trabajos con sede local. De los dos proyectos en donde emerge el cine de Colombia, uno de ellos tiene sede allí. Por último, tanto la cinematografía de México como la de Brasil figuran conjuntamente con otro cine en un proyecto por lado; ambos radicados en el país. Ahora bien, en esta sección habría que señalar de manera diferenciada una serie de proyectos que no analizan la relación entre dos cinematografías nacionales sino que ponen el foco de atención en el cine latinoamericano de forma global. De las diez investigaciones registradas, seis provienen de Brasil, tres de Argentina y una de Austria.

TABLA 1

\begin{tabular}{|l|l|l|l|l|}
\hline Cinematografía & \multicolumn{2}{|l|}{ En soledad } & \multicolumn{2}{l|}{ En articulación con otros cines } \\
\hline & Sede local & Sede externa & Sede local & Sede externa \\
\hline argentina & 4 proyectos & 1 proyecto & --- & 2 proyectos (a,b) \\
\hline boliviana & 1 proyecto & 1 proyecto & --- & --- \\
\hline brasileña & 1 proyecto & --- & 1 proyecto (a) & --- \\
\hline chilena & 2 proyectos & 2 proyectos & --- & --- \\
\hline colombiana & 1 proyecto & --- & 1 proyecto (b) & 1 proyecto (c) \\
\hline dominicana & 1 proyecto & --- & --- & --- \\
\hline mexicana & 3 proyectos & 1 proyecto & 1 proyecto (c) & --- \\
\hline venezolana & 6 proyectos & --- & --- & --- \\
\hline latinoamericana & 10 proyectos & & \\
\hline
\end{tabular}

En suma, algunas conclusiones preliminares pueden extraerse de esta parte del informe. Excluyendo a los trabajos enfocados en el cine de la región en su conjunto que como explicamos meren un comentario singular-, de los otros veintisiete proyectos veinticuatro se concentran en una sola cinematografía y diecinueve están radicados en el país asido como objeto de estudio. Estos números marcarían, por un lado, una leve tendencia a favor del interés acerca de los cines nacionales y, por el otro, una alta conexión entre la pertenencia institucional y el origen de la cinematografía escogida. No obstante, la observación sobre los vínculos entre los diversos cines de América Latina 
ocupa un lugar no menos importante. Aquello que sobresale, claro está, viene de la mano del creciente incentivo en el análisis del cine latinoamericano en tanto fenómeno de índole regional. De todas formas, la metodología del estudio comparado es una perspectiva que ha calado hondo en la investigación en torno al cine de América Latina. ${ }^{6}$ Por otra parte, podemos corroborar la heterogeneidad en el planteo de predominancia y origen de las cinematografías abordadas. Los cines de Argentina, Chile, México y Venezuela son los que más veces afloran, aunque Brasil presenta un alto grado de filiación institucional. Chile evidencia una gran variabilidad en el origen de los trabajos, mientras que el caso de Venezuela resulta peculiar. Una hipótesis con respecto a la existencia de este corpus de proyectos concentrados exclusivamente en este cine, originados en el propio país, podría ser el gran desconocimiento que presenta la cinematografía venezolana en el resto del continente, en relación a otros cines que han forjado una tradición cinematográfica con mayor sustento en el tiempo.

Desde otro ángulo, vale hacer un señalamiento acerca de las periodizaciones que dichos proyectos ostentan. Si bien el arco temporal de una investigación se halla estrechamente vinculado con la problemática analizada, los diferentes cortes sincrónicos en el eje de la historia del séptimo arte que estos trabajos proponen dan cuenta por sí solos de ciertas tendencias en la investigación actual sobre el cine latinoamericano. En principio, hay una serie de proyectos que no contemplan periodización alguna, ya sea por abordar al cine desde una disciplina distinta a la histórica - el cine como herramienta pedagógica, por ejemplo - o por estudiar tanto casos aislados como conceptualizaciones extremadamente generales, colocando el peso en otras variables. Luego, el recorrido temporal es bastante heterogéneo, y esto se debe a las especificidades y particularidades que ha desarrollado el cine al interior de la región. Mientras que en algunas cinematografías es posible hablar de un cine clásico - esto se ve reflejado en proyectos que analizan solamente la década del treinta, los años cuarenta y cincuenta, y el período del cuarenta al sessenta - y un cine moderno - trabajos que abarcan el tándem cincuenta/sesenta y sesenta/setenta -, existen otras investigaciones que exhiben límites temporales más abarcativos, transversales y poco tradicionales - el énfasis puesto en la curva del veinte al sesenta, del treinta al setenta o del sesenta al noventa. Sobresalen asimismo los proyectos enfocados en las décadas del setenta al

\footnotetext{
${ }^{6}$ Dicho método de análisis no es novedoso dentro del campo de estudios sobre el cine de la región, aunque este se ha intensificado en los últimos veinte años. Lo que sí ha variado en el tiempo es el tipo de comparación establecida. Pioneros como Alfonso Gumucio Dagron y Guy Hennebelle (1981), Julianne Burton (1986), Peter Schumann (1987), John King (1990), entre otros, utilizaban la comparativa en la introducción para luego efectuar un análisis dentro de los límites de los territorios nacionales. Otros investigadores como Zuzana Pick (1993) y Paulo Antonio Paranaguá (2003) plantearon ejes transversales de comparación. Es dentro de esta línea que se ubican los proyectos de RICiLa que asumen una metodología comparada.
} 
ochenta y del setenta al noventa, relacionados principalmente con las etapas dictatoriales y posdictatoriales acaecidas en gran parte de Latinoamérica. Finalmente, dos datos llaman la atención: la escasa cantidad de trabajos destinados al análisis de la época silente $^{7}$ y la supremacía de la etapa contemporánea. Trece proyectos están focalizados en el cine del nuevo milenio, lo cual marca en cierta forma una predilección por el estudio del fenómeno cinematográfico actual.

\section{El cine latinoamericano en tanto objeto y campo de estudio consolidado: ¿Cuáles son los temas de análisis y qué enfoques de aproximación imperan?}

Una multiplicidad de criterios podrían utilizarse en pos de organizar esta sección central del informe. En este caso hemos escogido recuperar los lineamientos que emergieron del análisis consumado en el apartado anterior con el propósito de mantener una cierta lógica de desarrollo y el interés de construir un camino orgánico que desemboque en una reflexión final, consecuente y productiva. En este sentido, comenzaremos estudiando de manera conjunta aquellos trabajos que contemplan una sola cinematografía, para proseguir luego con los proyectos que abordan de modo comparado dos cines nacionales y concluir con las investigaciones regionales acerca del cine latinoamericano. De esta forma, intentaremos establecer similitudes y diferencias en torno a los enfoques de aproximación al interior de cada bloque. Dentro de los mismos surgirán, en tanto rangos de contraste, diversos modos de vincular a los distintos trabajos.

De acuerdo a lo señalado precedentemente, los proyectos que se enfocan en una cinematografía nacional predominan por sobre el resto. En primer lugar, reparamos en seis trabajos que se nuclean en torno a la exploración del cine contemporáneo en un país determinado. Las investigaciones de Malena Verardi y María Celina Ibazeta adoptan la perspectiva del análisis de obra. La primera, titulada El documental como forma en el cine argentino contemporáneo. Usos y productividad en relación con la escena socio-cultural, piensa el documental argentino actual desde un enfoque narratológico, a partir de las categorías formales del tiempo y del espacio. La segunda efectúa un análisis estético y discursivo de los documentales contemporáneos mexicanos sobre la migración, focalizando en el recurso del montaje y la utilización del sonido. Ambos trabajos, centrados en el modelo documental, mantienen una relación

\footnotetext{
${ }^{7}$ Esta información resulta sorpresiva puesto que ha aumentado en los últimos años el estudio académico sobre el cine latinoamericano de este período. Dos hitos avalan esta apreciación: la publicación digital Vivomatografías. Revista de estudios sobre precine y cine silente en Latinoamérica surgida en 2015 y la reciente creación de la Asociación de Estudios sobre Precine y Cine Silente Latinoamericano (PRECILA).
} 
estrecha con el contexto de producción y la serie social. Por su parte, los proyectos de Lucía Hinojosa Córdova, Vania Barraza, Sebastián Morales Escoffier y Vania Berger plantean un acercamiento global a la institución-cine de cuatro naciones diferentes: México, Chile, Bolivia y Argentina. El primero observa la reestructuración de la industria mexicana a partir de las políticas económicas neoliberales y a través de una orientación eminentemente descriptiva. El segundo también tiene en cuenta el impacto del neoliberalismo, en este caso, en relación al cine chileno contemporáneo de la postransición aunque desde una perspectiva socio-crítica. El tercero indaga sobre los modos de producción, distribución y consumo del cine digital realizado en La Paz en los últimos doce años. El cuarto tiene como objetivo dilucidar el funcionamiento de las coproducciones argentinas contemporáneas, colocando el eje de atención en la política de subvenciones junto a la producción y distribución por medio de festivales. Este último estudio grupal, si bien se posiciona desde una sola cinematografía, sugiere asimismo un punto de vista transnacional.

En segunda instancia encontramos cuatro proyectos dedicados a la problemática del cine y los procesos dictatoriales en los años setenta y ochenta en Argentina, Bolivia y Chile. El trabajo de Ana Laura Lusnich, Significación histórica de las ficciones hermético-metafóricas realizadas en Argentina en años de la última dictadura militar: estudio de la recepción crítica y del comportamiento de las audiencias, y el de María Aimaretti, Emergencia democrática y esfera ciudadana: resistencia no violenta, duelo y creatividad en el campo audiovisual boliviano (1977-1989). Experiencias culturales de tramitación del dolor y la palabra postergada, intentan examinar ciertas estrategias de resistencia - implícitas o expliciticas - del cine frente a la dictadura militar en los respectivos países y su recepción tanto en el contexto de producción como en los años posteriores durante la transición democrática. Desde un punto de vista totalmente diferente, Luis Horta Canales se detiene en el análisis fílmico del aspecto estético y del lenguaje en los films producidos y fomentados por la dictadura militar en Chile. Finalmente, la investigación de José Miguel Palacios explora, a través de la filosofía de la historia y de los estudios culturales, el cine chileno del exilio luego del golpe militar de 1973, comprendido como un cine transnacional. Al igual que el último trabajo del grupo anterior, este complejiza la noción de un cine nacional anclado en un solo territorio.

Por otro lado, es posible congregar bajo una misma línea a tres proyectos que organizan la pesquisa alrededor del análisis de un film emblema y su productividad al interior de una cinematografía. Este es el caso de Ricardo Azuaga, quien examina el film Pandemónium, la capital del infierno (1997) como una obra de ruptura dentro de la filmografía del cineasta Román Chalbaud y en marcada conexión con el contexto social de Venezuela a fines de los años noventa. El trabajo de Carlos Delgado Díaz también 
está dirigido al reparo del cine en Venezuela. En esta oportunidad la propuesta consiste en realizar un análisis historiográfico y analítico acerca de la primera película sonora venezolana, La Venus de Nácar (Efraín Gómez, 1932), como puntapié inicial para la instauración de un nuevo paradigma en el cine nacional. Con implicancias análogas, aunque centrado en el cine mexicano de los primeros tiempos, el trabajo de Kassandra Sifuentes efectúa un estudio en torno a la primera película de Monterrey y su impacto en el desarrollo de una proto-industria cinematográfica.

En otra senda, tres trabajos perfilan sus investigaciones en desarrollos cinematográficos nacionales extendidos en el tiempo. Por ejemplo, Ana Laura Lusnich dirige el proyecto colectivo denominado Cartografía y estudio histórico de los procesos cinematográficos en Argentina (1896-2016), el cual propone llevar a cabo un estudio sistematizado de la producción, la distribución y la exhibición del cine en el país -fuera del área metropolitana- desde los orígenes hasta la actualidad. El programa de Kassandra Sifuentes, Implicaciones sociales y culturales relativos al desarrollo del cine en Monterrey, 1928-1960, se erige como una continuación de su anterior proyecto con el propósito de observar las transformaciones del cine de Monterrey en relación a los cambios experimentados dentro de la industria del cine y en relación al empresariado durante las tres décadas posteriores a la llegada del sonido. Por último, Luis Horta Canales y su grupo sitúan el eje de reflexión en el papel que desempeñó la Universidad de Chile en el desarrollo del cine chileno desde 1929 hasta el desmantelamiento de la misma en 1973, producto del golpe militar. El binomio cine-educación atraviesa dicho trabajo.

Asimismo, podemos señalar ciertos proyectos que adoptan como hilo conductor enfoques singulares de tradición consolidada: los estudios de recepción, la puesta en valor patrimonial y la perspectiva de género. En relación al primero, Jose Alirio Peña Zerpa procura examinar las experiencias de los usuarios para con el cine Urdaneta en Venezuela, entendido como espacio fijo queer. En cuanto al segundo, Rosanna Solano tampoco refiere su estudio estrictamente a la cinematografía de su país sino que, en este caso, pretende llevar adelante un análisis histórico de la Cinemateca Dominicana en tanto organismo de salvaguarda y difusión del material audiovisual nacional e internacional. El tercer trabajo, correspondiente a la tesis doctoral de Julia Kratje, tiene como finalidad aproximarse al cine de ficción argentino contemporáneo desde la perspectiva de género para analizar los films centrados en la vida cotidiana de las mujeres tomando los aportes de la teoría feminista y los estudios vinculados al ocio.

Desde otro ángulo, dos investigaciones ponen un marcado énfasis en la relación del cine con la historia, a través de los films de compilación en Brasil y del documental en Venezuela. Se trata de los trabajos de Eduardo Victorio Morettin y María Gabriela 
Colmenares España. En el primero, rotulado Imagem-documento, imagem-monumento e cinema de arquivo: cruzamentos históricos em tempos de formação, engajamento e propaganda, el autor analiza los films de compilación en Brasil entre las décadas del veinte y del cuarenta con el interés de reflexionar sobre el carácter de documento y monumento del cine en un contexto de polarización ideológica. En el segundo, la responsable de la tesis doctoral titulada Venezuela en marcha: imaginario social y representaciones de la nación moderna en los documentales de la industria petrolera venezolana (1947-1968) realiza un análisis textual de un corpus de documentales institucionales ligados a la industria del petróleo para observar la representación social de la nación moderna haciendo hincapié en dos modelos particulares: la democracia liberal y el despotismo ilustrado de la dictadura militar.

En otro orden de cosas, dos proyectos centrados en el cine venezolano toman a los festivales como objeto de estudio, atravesados por un enfoque ambientalista. Claritza Peña Zerpa participa de este trabajo colectivo que promueve examinar el aspecto narrativo de los cortometrajes del denominado cine verde dentro del festival local FESTIVERD para colocar el acento en la función didáctica de dichas producciones audiovisuales. En sintonía con el proyecto anterior, Mixzaida Yelitza Peña Zerpa pone el foco de atención en el activismo ambiental en los festivales de cine verde venezolanos aunque para trascender este ámbito y trazar finalmente un estado de la cuestión del activismo ambiental en todo el cine venezolano, tratando de identificar usos y funciones de la producción audiovisual con dichos fines.

Finalmente, el último proyecto que contempla el estudio de una sola cinematografía es el que coordina Álvaro Andrés Villegas Velez, el cual combina un análisis textual de un corpus amplio de películas y un examen contextual basado en políticas de fomento para abordar un período y un país hasta el momento ausentes en el relevamiento de este apartado: el cine moderno colombiano. Este trabajo se propone revisar la producción que la historiografía y la crítica han catalogado como nuevo cine colombiano, renovando tanto el corpus como la metodología de acercamiento a los films.

Los tres proyectos que abordan conjuntamente dos cinematografías latinoamericanas están nucleados en el cine contemporáneo. La investigación de Natalia Christofoletti Barrenha, denominada Horror e fantasmas no cinema contemporâneo da Argentina e do Brasil, pretende examinar la incorporación de temáticas del horror como subtexto en producciones argentinas y brasileñas del período actual que no necesariamente logran insertarse completamente dentro de dicho género. Ahora bien, los otros dos trabajos ubican el núcleo de interés en el método comparado y entienden la comparativa entre dos cinematografías como parte de un proceso regional más amplio, lo cual los sitúa en la puerta hacia los estudios sobre cine latinoamericano de 
forma global. José Gabriel Cristancho Altuzarra aborda los procesos de memoria en el cine argentino y colombiano de principios del siglo XXI en tanto fenómeno compartido por grupos políticos de oposición en gran parte de América Latina. Esta tesis doctoral titulada Tigres de papel, recuerdos de película. Memoria, oposición y subjetivación política en el cine argentino y colombiano destina un capítulo para cada país en donde se analizan las especificidades locales para luego reflexionar en un capítulo aparte sobre las recurrencias, confluencias y diferencias que pueden vislumbrarse en torno a la construcción de las políticas de la memoria en ambas cinematografías. Por su parte, Susadny González Rodríguez propone un análisis comparado de las dinámicas de distribución y exhibición contemporáneas en Colombia y Chile, a la luz de las políticas cinematográficas de fomento y en relación a las transformaciones legislativas experimentadas por la región a comienzos del siglo XXI.

Finalmente, repasamos aquellos trabajos que estudian comparadamente tres 0 más cinematografías como parte de procesos representativos de un fenómeno global y los que no hacen discriminación de países para enfocarse en la región de forma integral. En algunos casos el método comparado trasciende sus confines hacia el campo de lo transnacional. ${ }^{8}$ Dentro de este último grupo podemos señalar los proyectos de Guilherme Maia y Vania Verger, los cuales buscan indagar en los elementos transnacionales de dos géneros cinematográficos específicos a lo largo y ancho de América Latina, sin distinción de nacionalidades ni períodos. En el primero, el objetivo de este trabajo grupal consiste en realizar una cartografía de los films musicales latinoamericanos de largometraje ficcionales y documentales para luego llevar a cabo un análisis tanto de las producciones como de la relación del texto con la serie social y encontrar parámetros comunes. En el segundo, el punto de atención está puesto en el análisis de las convenciones - patrones narrativos y visuales - de las road movies y sus rasgos transnacionales en films producidos por directores de Latinoamérica. Si bien el proyecto pretende abocarse a las cinematografías nacionales menos consideradas, el trabajo abarca toda la región.

Por otra parte, dos investigaciones concentradas en el cine contemporáneo también se aproximan a la región de manera integral, en este caso de la mano de las coproducciones. El trabajo en coautoría de Marina Moguillansky y Leandro González tiene como aspiración la construcción de una cartografía de las coproducciones cinematográficas en América Latina entre 1995 y 2015. Esta práctica es entendida como

\footnotetext{
${ }^{8}$ Es decir que, en lugar de poner en relación dos expresiones particulares para observar semejanzas y divergencias se procura "ofrecer una interpretación productiva y concreta de las conexiones que se establecen entre lo local y lo global, o entre lo nacional y lo transnacional" (LUSNICH, 2014: 106).
} 
un espacio cinematográfico supranacional y el proyecto busca estudiar su evolución así como desentrañar las combinaciones más comunes y el origen de los fondos utilizados. A su vez, Rosangela Fachel postula un nuevo enfoque sobre los cines contemporáneos del Mercosur desde una perspectiva que combina el análisis fílmico junto con la aplicación de diversas teorías en torno a cuestiones económicas, políticas y sociales. En definitiva, la autora toma las coproducciones como un elemento transnacional que colabora en la conformación de una identidad regional en un contexto globalizado de hegemonía hollywoodense.

En otra línea, dos trabajos se acercan al cine moderno en América Latina a partir de enfoques disímiles. Por un lado, Javier Cossalter explora la productividad del cortometraje en Latinoamérica durante las fases de gestación y desarrollo de la modernidad cinematográfica. Allí se lleva adelante un estudio comparado con la intención de descubrir los componentes estéticos renovadores del film breve en la región. Por el otro, Marina Cavalcanti Tedesco repara en las mutaciones del campo audiovisual moderno en relación a la construcción de la imagen de los hombres y las mujeres a través de una perspectiva de género, teniendo en cuenta las transformaciones sociales acaecidas en dicho período.

De manera singular subrayamos el trabajo de Marco Túlio Ulhôa, puesto que es el único proyecto que propone vincular el cine con otra disciplina artística. El proyecto titulado A imagem como a última das histórias possíveis: José Lezama Lima e o anacronismo das imagens em movimento se propone analizar la influencia de la literatura hispano-americana en el cine de América Latina durante las décadas de 1950 y 1980 a partir de la producción artística e intelectual del escritor cubano José Lezama Lima.

Por último, apuntamos sobre tres investigaciones que examinan de forma comparada, en tres o más cines nacionales, un género cinematográfico particular de gran productividad en el período contemplado: la comedia en el cine clásico, el cine histórico en los años sesenta y setenta, el documental político en la actualidad. En relación al primer tópico, Alejandro Kelly Hopfenblatt estudia la comedia sofisticada entre 1945 y 1956 en los cines de Argentina, México, Chile y Venezuela desde una perspectiva transnacional y en tanto estrategia de mediación del proceso modernizador. En cuanto al segundo tema, Ignacio Del Valle Dávila sugiere el análisis de la representación de la independencia en largometrajes de ficción en los años sesenta y setenta en Argentina, Brasil y Cuba, en un contexto de emergencia del cine histórico ligado a procesos de emancipación nacional. El tercer proyecto, a cargo de Fernando Seliprandy, promete el estudio comparado de tres documentales políticos contemporáneos hechos por hijos de 
ex-militantes del partido político de izquierda en Argentina, Brasil y Chile en un momento de auge de esta tendencia de recuperación de la memoria en torno al pasado reciente.

En último lugar debemos destacar dos proyectos que quedan fuera de todas las líneas de agrupamiento esbozadas, ya que se trata de trabajos que no contemplan ninguna cinematografía en especial pero que se estima tienen la intención de ponerse en práctica alrededor del cine latinoamericano. En el primero de ellos, Leandro González y su grupo de investigación proveniente de Argentina anhelan explorar formas alternativas de producción y consumo audiovisual atravesadas por las políticas de innovación en los sistemas de producción. En el segundo, Claritza Peña Zerpa, oriunda de Venezuela, elabora un plan de capacitación docente para el empleo del cine como herramienta pedagógica en la educación primaria.

Como coda de este apartado resulta pertinente hacer un señalamiento sobre ciertos autores y referencias bibliográficas comunes utilizadas para desarrollar estos proyectos, las cuales sustentan algunas de las problemáticas y de los enfoques revisitados. En principio, es posible distinguir investigaciones medulares acerca de cinematografías nacionales tanto en términos generales como en relación a períodos puntuales. En cuanto a esta última línea sobresalen los libros de Ana Amado (2009) sobre el cine argentino entre la transición democrática y la contemporaneidad dedicado a la recuperación de la memoria del pasado reciente - y de Gonzalo Aguilar (2006) nucleado en el nuevo cine argentino-, y los textos de Lucila Hinojosa Córdova (2010, 2012) - sobre el cine mexicano contemporáneo. En derredor al estudio de determinados cines a nivel global mencionamos los trabajos de Ambretta Marrosu (1988) - sobre la cinematografía venezolana - y de Lucía Hinojosa Córdova (2003) - en torno al cine mexicano. Ahora bien, particular atención merecen aquellos escritos fundamentales que ponen el centro de interés en el cine latinoamericano y su dimensión regional/transnacional. La primera fuente citada y obra de consulta obligada para los proyectos enfocados en el cine de América Latina es el libro de Paulo Antonio Paranaguá (2003), Tradición y modernidad en el cine de América Latina. Los volúmenes de Zuzanna Pick (1993) y John King (1994) también se conformaron en tanto obras destacadas, aunque como hemos comentado con anterioridad, disponen de otra perspectiva comparatista. Asimismo, libros contemporáneos de investigadores que forman parte de RICiLa y que participan de la misma con proyectos propios se han convertido actualmente en textos canónicos en torno a esta temática. Este es el caso de El Nuevo Cine Latinoamericano y su dimensión continental. Regionalismo e integración cinematográfica (FLORES, 2013) y Cámaras en trance. El nuevo cine latinoamericano, un proyecto cinematográfico subcontinental (DEL VALLE DÁVILA, 2014). Finalmente, se destacan una serie de estudios sobre cines transnacionales, tanto en términos 
teóricos como en su aplicación al cine latinoamericano. Entre los primeros se vislumbran Ios textos fundacionales de Elizabeth Ezra y Terry Rowden (2006), y Nataša Ďurovičová y Kathleen Newman (2010). Entre los segundos nombramos, entre otros, los trabajos de Libia Villazana (2009), Alfonso Gumucio Dagron (2012) y Minerva Campos (2013).

\section{Reflexiones finales}

A lo largo del presente trabajo hemos intentado dar cuenta de las problemáticas abordadas en los proyectos de los integrantes de la Red de Investigadores sobre Cine Latinoamericano y su contexto de producción. Resulta indispensable enfatizar que este mapeo no alberga a todas las investigaciones sobre cine latinoamericano del conjunto de los miembros de RICiLa, ni mucho menos pretende ser representativo de la totalidad de estudios existentes sobre dicha temática. No obstante, el relevamiento efectuado y su puesta en relación a partir de la metodología del análisis comparado permiten vislumbrar algunas claves en torno al panorama actual sobre el campo de estudios en derredor a los cines de la región. A ocho años de la creación de la Red, luego del fortalecimiento de los vínculos entre sus miembros y el progresivo crecimiento de los escritos sobre el cine de América Latina, era necesario tomar conocimiento de cómo se desarrollan estas investigaciones y cuáles son las inquietudes que motivan dichos planteamientos. Este es el lugar de enunciación asido para encarar el informe y de aquí se desprende su pertinencia.

En primer lugar, hemos podido observar que la universidad estatal y pública se erige como el principal entorno de fomento de proyectos dedicados a la investigación en cine latinoamericano. La diversidad de facultades, departamentos y centros universitarios hallados marca el interés creciente y sostenido del campo académico en la pesquisa de este fenómeno. En este punto es posible añadir un dato de relevancia que se relaciona con la procedencia institucional. De la serie global de trabajos contabilizamos trece investigaciones de posgrado - ocho de las cuales se corresponden con tesis finalizadas o proyectos de doctorado en curso -, lo que indica el papel fundamental de la universidad en la formación de jóvenes investigadores y el sustento brindado para el desarrollo de temáticas afines a los problemas ligados al séptimo arte. Por otra parte, entidades emplazadas fuera del ámbito educativo formal, aunque dedicadas exclusivamente el campo cinematográfico, se presentan asimismo como promotoras de los estudios sobre cine. Otro detalle que resulta interesante es que, si bien la mayoría de los proyectos son de carácter individual, encontramos al menos ocho trabajos puestos en marcha de manera colectiva - ya sean de corte académico o no -, hecho que demuestra la existencia de grupos de investigación -formados o en vías de consolidación - que colocan sus esfuerzos en el análisis del cine latinoamericano. 
En segunda instancia, hemos advertido que predominan los proyectos enfocados en una sola cinematografía nacional y que gran parte de estos pertenecen a investigadores que están radicados en el país que toman por objeto de estudio. Esto manifiesta que, a pesar de la globalización, el interés por lo local sigue teniendo un peso sustancial. Sin embargo, no se puede dejar de señalar una tendencia creciente por la exploración de lo extranjero y una predilección por el estudio comparado entre dos o más cinematografías. ${ }^{9}$ Ahora bien, mención aparte merecieron aquellos proyectos que se aproximaban al cine latinoamericano en su dimensión regional, articulando en algunos casos un componente transnacional. Las referencias bibliográficas citadas en derredor a este aspecto dejan entrever que el análisis comparativo y el enfoque transnacional no sólo se erigen como métodos eficaces de acercamiento - la recurrencia de autores canónicos como Paulo Antonio Paranaguá-, sino que también se constituyen en objetos de estudio en sí mismos - escritos coetáneos de investigadores jóvenes como Silvana Flores e Ignacio Del Valle Dávila evidencian esta apreciación. Es quizás esta orientación uno de los rumbos posibles por donde transite el devenir de los estudios acerca del cine en la región. En cuanto a los períodos históricos contemplados en los trabajos la principal nota a subrayar es la predominancia del cine contemporáneo por sobre cualquier otro marco temporal, aunque tal como hemos discernido - en mayor o menor medida - las diferentes etapas en la historia del cine han tenido su lugar.

En relación al apartado central del ensayo, la primera conclusión que aflora sobre las problemáticas escogidas es la presencia de una heterogeneidad de variantes temáticas y de perspectivas de análisis. No obstante, y a pesar de las singularidades, hemos logrado distinguir algunos patrones comunes. En principio, el análisis textual de un film o un corpus fílmico en pos de dar cuenta de los elementos estéticos que afloran en una fase, corriente o cinematografía es una de las modalidades privilegiadas de análisis. En ciertos casos, este enfoque semiótico se complementa con un reparo consciente sobre el contexto social de producción. Por otra parte, la exanimación de la institución-cine es otro de los tópicos más trabajados en los proyectos. Estos se concentran alrededor de la preocupación por conocer el desarrollo de las instancias de producción, distribución y exhibición de una cinematografía en una etapa determinada. Asimismo, los estudios de recepción aparecen ligados a contextos dictatoriales o a estadíos de recuperación de la memoria, problemática que ocupa un espacio importante en las investigaciones de RICiLa. En otra línea, también hemos encontrado una serie de

\footnotetext{
${ }^{9}$ En torno a esta directriz se recomienda enfáticamente la lectura de Lusnich (2011), en donde la autora traza un recorrido histórico sobre los estudios comparados sobre el cine de América Latina y propone nuevas conceptualizaciones para comprender los distintos abordajes aludidos con anterioridad en este trabajo.
} 
trabajos que ponen el foco de atención en procesos desarrollados a lo largo del tiempo, rompiendo las variables tradicionales de periodización de la historia del cine. Por su parte, la exploración de géneros cinematográficos como la comedia, el cine histórico y el documental político se han hecho presentes. A su vez, perspectivas de abordaje como la de género o el perfil patrimonial tuvieron eco en algunos proyectos. Por último, fenómenos como las coproducciones y los festivales son tópicos cuyo interés está en auge. En este punto el enfoque transnacional ha tenido mayor pregnancia, aunque como hemos señalado, este se ha convertido en un campo de estudios singular, acompañando objetos particulares como por ejemplo las road movies y los musicales.

En suma, el objetivo de este escrito es dar a conocer la producción de investigación en torno al cine de la región de los miembros de la Red de Investigadores sobre Cine Latinoamericano, con el propósito de visibilizar las temáticas predominantes, los métodos y perspectivas de análisis, hacer un balance del desarrollo actual de los estudios sobre el cine de América Latina y plantear algunos núcleos de fortalecimiento que pueden generar líneas de trabajo a futuro. Finalmente, el anhelo principal de este trabajo es incentivar aún más la pesquisa en torno a nuestros cines e invitar a los investigadores de todo el mundo a formar parte de RICiLa.

\section{Referencias bibliográficas}

AGUILAR, G. Otros mundos. Un ensayo sobre el nuevo cine argentino. Buenos Aires: Santiago Arcos, 2006.

AMADO, A. La imagen justa. Cine argentino y política (1980-2007). Buenos Aires: Colihue, 2009.

BURTON, J. Cinema and social change in Latin America: conversation with filmmakers. Texas: University of Texas Press, 1986.

CAMPOS, M. "La América Latina de 'Cine en construcción'. Implicaciones del apoyo económico de los festivales internacionales". Archivos de la Filmoteca. 71, 2013,. 13-26. Disponible en: http://www.archivosdelafilmoteca.com/index.php/archivos/article/view/392. Acceso el: 5 de Septiembre de 2019.

DEL VALLE DÁVILA, I. Cámaras en trance. El nuevo cine latinoamericano, un proyecto cinematográfico subcontinental. Santiago de Chile: Editorial Cuarto Propio, 2014.

ĎUROVIČOVÁ, N. \& Newman, K. World Cinemas. Transnational Perspectives. New York: Routledge, 2010.

EZRA, E. \& ROWDEN, T. Transnational Cinema. The Film Reader. New York: Routledge, 2006.

FLORES, S. El Nuevo Cine Latinoamericano y su dimensión continental. Regionalismo e integración cinematográfica. Buenos Aires: Imago Mundi, 2013. 
GUMUCIO DAGRON, A. (Coord.). Estudio de Experiencias del Cine y el Audiovisual Comunitarios en América Latina y el Caribe. La Habana: Cuadernos de Estudios. Fundación del Nuevo Cine Latinoamericano, 2012.

HENNEBELLE, G. \& Gumucio Dagron, A. Les Cinémas de l'Amérique Latine d'Aujourd'hui. París: Lherminier, 1981.

HINOJOSA Córdova, L. El cine mexicano: la identidad cultural y nacional. Nuevo León: Editorial Trillas, 2003.

"Cine transnacional y espectadores globales: consumo y recepción de películas mexicanas en Monterrey, México". In: NIETO, Jorge (Ed.). Sociedad, desarrollo y movilidad en comunicación. Tampico: Universidad Autónoma de Tamaulipas, 2010, 310-326.

(2012). "Economía política del cine mexicano: oferta y consumo de películas nacionales en Monterrey, México (2001-2010)". Eptic Online, Revista Electrónica Internacional de Economía Política de las Tecnologías de la Información y Comunicación, 14(3), 2012,. $1-21 . \quad$ Disponible en: https://seer.ufs.br/index.php/eptic/issue/view/62. Acceso el: 15 de Marzo de 2020.

KING, J. El carrete mágico: Una historia del cine latinoamericano. Bogotá: TM Editores, 1990.

LUSNICH, A. L. "Pasado y presente de los estudios comparados sobre cine latinoamericano". Comunicación y Medios, 24, 2011,. 25-42. Disponible en: DOI: 10.5354/0719-1529.2012.19892. Acceso el: 2 de Julio de 2019.

"Del comparatismo al transnacionalismo. Bases para un estudio del cine argentino y mexicano del período clásico-industrial". TOMA UNO, 3, 2014, 99-110. Disponible en: https://revistas.unc.edu.ar/index.php/toma1/article/view/9294. Acceso el: 20 de Febrero de 2020.

MARROSU, A. "Periodización para una historia del cine venezolano (Una hipótesis)". Anuario ININCO, 1, 1988,. 7-45. Disponible en: http://190.169.94.12/ojs/index.php/rev ai/issue/view/1334/showToc. Acceso el: 20 de Febrero de 2020.

PARANAGUÁ, P. A. Tradición y modernidad en el cine de América Latina. Madrid: Fondo de Cultura Económica, 2003.

PICK, Z. The New Latin American Cinema. A Continental Project. Austin: University of Texas Press, 1993.

SCHUMANN, P. Historia del cine latinoamericano. Buenos Aires: Legasa, 1987.

VILLAZANA, L. Transnational financial structures in the cinema of Latin America: Programa Ibermedia in study. Berlin: VWM Verlag, 2009. 